TRANSACTIONS OF THE

AMERICAN MATHEMATICAL SOCIETY

Volume 356, Number 11, Pages 4493-4518

S 0002-9947(04)03397-5

Article electronically published on January 13, 2004

\title{
VARIATION INEQUALITIES FOR THE FEJÉR AND POISSON KERNELS
}

\author{
ROGER L. JONES AND GANG WANG
}

\begin{abstract}
In this paper we show that the $\varrho$-th order variation operator, for both the Fejér and Poisson kernels, are bounded from $L^{p}$ to $L^{p}, 1<p<\infty$, when $\varrho>2$. Counterexamples are given if $\varrho=2$.
\end{abstract}

\section{INTRODUCTION}

Let $\mathbb{T}$ denote the interval $[-\pi, \pi)$, thought of as the unit circle, with normalized Lebesgue measure. For a function $f \in L^{1}(\mathbb{T})$, we have

$$
\hat{f}(n)=\frac{1}{2 \pi} \int_{-\pi}^{\pi} f(t) e^{i n x} d x .
$$

We denote by $K_{n}(x)$ the Fejér kernel given by

$$
K_{n}(x)=\sum_{j=-n}^{n}\left(1-\frac{|j|}{n+1}\right) e^{-i j x}=\frac{1}{n+1}\left(\frac{\sin \frac{n+1}{2} x}{\sin \frac{x}{2}}\right)^{2} .
$$

We let $\sigma_{n} f(x)=\left(K_{n} * f\right)(x)$, where as usual, $f * g$ denotes the convolution of $f$ and $g$.

We will also consider the Poisson kernel, which for $0 \leq r<1$ is given by

$$
P_{r}(x)=1+2 \sum_{j=1}^{\infty} r^{j} \cos j x=\frac{1-r^{2}}{1-2 r \cos x+r^{2}} .
$$

The operators associated with these convolution kernels play a very fundamental role in harmonic analysis, and understanding their behavior has been the subject of considerable research. In this paper we study the degree of oscillation of the family of operators associated with each of these kernels as they approach their limit. We do this both by looking at the maximum $\varrho$-variation (defined below) and by counting the number of times that the family of operators takes a jump of a certain size. (The number of these jumps can be related to the number of upcrossings of the family of operators.) Our method of proof involves relating these operators, via square functions, to the dyadic differentiation operator. (See 7] for an interesting historical discussion of the role of square functions in classical

Received by the editors August 17, 2001 and, in revised form, May 13, 2003.

2000 Mathematics Subject Classification. Primary 42A24; Secondary 26D05.

Key words and phrases. Fejér kernel, Poisson kernel, square functions, variation, jump inequalities.

The first aurthor was partially supported by a grant from the DePaul University Liberal Art and Science research program.

The second author was partially supported by NSF grant DMS-0071759. 
analysis.) The dyadic differentiation operator can in turn be related to a dyadic martingale. For the martingale (and hence for the differentiation operator) we can use already established results for the $\varrho$-variation and the number of jumps (see [2]). The connection of these operators to the differentiation operator is of independent interest since it shows clearly why many results are true for operators considered in Fourier analysis.

We define the $\varrho$-variation operator for the Fejér and Poisson kernels as follows.

Definition 1.1. For the Fejér kernel,

$$
\sigma_{n} f(x)=\left(K_{n} * f\right)(x)=\frac{1}{2 \pi} \int_{-\pi}^{\pi} f(x-t) K(t) d t,
$$

we define the $\varrho$-variation operator

$$
V_{\varrho}(K f)(x)=\sup _{\left(n_{j}\right)}\left(\sum_{j=1}^{\infty}\left|\sigma_{n_{j}} f(x)-\sigma_{n_{j+1}} f(x)\right|^{\varrho}\right)^{\frac{1}{\varrho}},
$$

where the supremum is taken over all increasing sequences $\left(n_{j}\right)$. For the Poisson kernel,

$$
P_{r} f(x)=\left(P_{r} * f\right)(x)=\frac{1}{2 \pi} \int_{-\pi}^{\pi} f(x-t) P_{r}(t) d t,
$$

we define the $\varrho$-variation operator

$$
V_{\varrho}(P f)(x)=\sup _{\left(s_{j}\right)}\left(\sum_{j=1}^{\infty}\left|P_{s_{j}} f(x)-P_{s_{j+1}} f(x)\right|^{\varrho}\right)^{\frac{1}{\varrho}},
$$

where the supremum is taken over all increasing sequences $\left(s_{j}\right)$, with $0 \leq s_{j}<1$ for each $j$.

We also define the $\lambda$-jump operator for the Fejér kernel as follows.

Definition 1.2. The $\lambda$-jump operator for the Fejér kernel will be denoted by $\Lambda(K, f, \lambda)(x)$ and is defined by

$$
\begin{array}{r}
\Lambda(K, f, \lambda)(x)=\sup \left\{n \in \mathbb{Z}^{+}: \text {there exist } s_{1}<t_{1} \leq s_{2}<t_{2}<\cdots \leq s_{n}<t_{n}\right. \\
\text { such that } \left.\left|\sigma_{s_{i}} f(x)-\sigma_{t_{i}} f(x)\right|>\lambda \text { for } i=1,2, \ldots, n\right\} .
\end{array}
$$

For the Poisson kernel the $\lambda$-jump operator $\Lambda(P, f, \lambda)(x)$ is defined in the analogous way.

Remark 1.3. If the $\lambda$-jump operators is finite a.e. for each choice of $\lambda>0$, then we must have a.e. convergence of our family of operators.

For example, with the Fejér kernel, note that finiteness of the $\lambda$-jump operator implies that for any given $\lambda$ the terms in the sequence $\left(\sigma_{n} f(x)\right)$ can differ by more than $\lambda$ only a finite number of times. After that finite number of terms all the remaining terms are within a band of thickness $2 \lambda$, and convergence follows by Cauchy's criterion.

The first issue to address regarding the $\varrho$-variation is that of measurability. In the case of the variation operator for the Fejér kernel, we can first take the supremum over all sequences bounded by $N$ for $N$ fixed. Then we have a supremum over only a finite number of sequences, and there is no problem with measurability. We can then let $N$ increase to infinity, and again, there is no problem with the measurability of this increasing family. For the variation of the Poisson kernel, we first consider 
sequences $\left(r_{j}\right)$ selected from the set $\left\{k / 2^{N}: k=0,1, \ldots, N-1\right\}$, and then let $N$ go to infinity. The "smoothness" of the Poisson kernel allows us to the pass to general increasing sequences.

Remark 1.4. If for some $\varrho<\infty$ the $\varrho$-variation is finite at a point $x$, then we have convergence at the point $x$.

To see this in the case of the Fejér kernel, assume that convergence fails. Then we know that there is an $\epsilon>0$ such that $\lim \sup \sigma_{n} f(x)-\liminf \sigma_{n} f(x)>\epsilon$. In this case we will show that we can select a sequence $\left(n_{k}\right)$ to make the variation infinite. First select $n_{1}$ so that $\sigma_{n_{1}} f(x)$ is close to $\lim \sup \sigma_{n} f(x)$. To be precise, select $n_{1}$ so that $\left|\lim \sup \sigma_{n} f(x)-\sigma_{n_{1}} f(x)\right|<\frac{\epsilon}{4}$. Next select $n_{2}>n_{1}$ such that $\sigma_{n_{2}} f(x)$ is close to $\liminf \sigma_{n} f(x)$, in particular, so that $\left|\liminf \sigma_{n} f(x)-\sigma_{n_{2}} f(x)\right|<\frac{\epsilon}{4}$. Then select $n_{3}>n_{2}$ so that $\sigma_{n_{3}} f(x)$ is close to the limsup $\sigma_{n} f(x)$, etc. In the end we have a sequence such that $\left|\sigma_{n_{k}} f(x)-\sigma_{n_{k+1}} f(x)\right|>\frac{\epsilon}{2}$ for each $k \geq 1$. Thus $V_{\varrho}(K f)(x) \geq\left(\sum_{k=1}^{\infty}\left(\frac{\epsilon}{2}\right)^{\varrho}\right)^{\frac{1}{\varrho}}=+\infty$.

We will prove the following four theorems.

Theorem 1.5. For $1<p<\infty$ and $\varrho>2$ we have $\left\|V_{\varrho}(K f)\right\|_{p} \leq c(p, \varrho)\|f\|_{p}$.

Theorem 1.6. For $1<p<\infty$ we have $\|\sqrt{\Lambda(K, f, \lambda)}\|_{p} \leq \frac{c(p)}{\lambda}\|f\|_{p}$.

Theorem 1.7. For $1<p<\infty$ and $\varrho>2$ we have $\left\|V_{\varrho}(P f)\right\|_{p} \leq c(p, \varrho)\|f\|_{p}$.

Theorem 1.8. For $1<p<\infty$ we have $\|\sqrt{\Lambda(P, f, \lambda)}\|_{p} \leq \frac{c(p)}{\lambda}\|f\|_{p}$.

It would be interesting to know if we can replace the Fejér or Poisson kernels by the Dirchlet kernel and still obtain a variation inequality or a $\lambda$-jump inequality. Since a norm inequality for either the variational operator or for the $\lambda$-jump operator associated with the Dirchlet kernel would imply Carleson's theorem on a.e. convergence of Fourier series, one would expect this to be hard. However as a consequence of the above results we can prove the following.

Theorem 1.9. Let $S_{n} f(x)$ denote the nth (symmetric) partial sum of a Fourier series and fix a lacunary sequence $\left(n_{k}\right)$. Then for $\varrho>2$ and $1<p<\infty$ the Q-variation operator associated with the sequence $\left(S_{n_{k}}\right)$ is a bounded operator on $L^{p}$.

Proof. The $\varrho$-variation operator satisfies a triangle inequality, and consequently $V_{\varrho}\left(S_{n_{k}} f\right)(x) \leq V_{\varrho}\left(\left(S_{n_{k}}-\sigma_{n_{k}}\right) f\right)(x)+V_{\varrho}\left(\sigma_{n_{k}} f\right)(x)$. The first term on the right is dominated by a constant multiple of $\left(\sum_{k=1}^{\infty}\left|S_{n_{k}} f(x)-\sigma_{n_{k}} f(x)\right|^{2}\right)^{\frac{1}{2}}$ (see (2.1) in Section 2), and this is shown to be bounded on all $L^{p}, 1<p<\infty$, by Zygmund [10], Chapter XV. The fact that the second term on the right is bounded is a consequence of Theorem 1.5

Remark 1.10. Since, as remarked above, finiteness of the $\varrho$-variation implies convergence, this result gives a proof that we have a.e. convergence of lacunary partial sums of a Fourier series.

Remark 1.11. Because the $\varrho$-variation operators satisfy a triangle inequality, we also have similar results for convolution with the de la Vallée Poussin kernel. This follows since the $n$th de la Vallée Poussin operator can be written as $2 \sigma_{2 n+1}-\sigma_{n}$. 
To see that $\varrho>2$ is required, we also state (and prove in Section 8 ) the following two negative results.

Theorem 1.12. There is a function $f \in L^{\infty}(\mathbb{T})$ such that $V_{2}(K f)(x)=\infty$ a.e.

Theorem 1.13. There is a function $f \in L^{\infty}(\mathbb{T})$ such that $V_{2}(P f)(x)=\infty$ a.e.

The proof of each of the above theorems will depend on comparison with another operator, which in turn can be compared with a martingale. In the case of a martingale the appropriate variation operator (for $\varrho>2$ ) has been studied by Lepingle [5] and shown to have bounds in $L^{p}, 1<p<\infty$. See also [2, where similar ideas were used to study the variation of ergodic averages.

Throughout the paper, $c$ and $C$, sometimes with additional parameters, will denote constants, but not necessarily the same constant from one occurrence to the next. Sometimes the values of $c$ or $C$ may differ even in the same line.

\section{Some NOTATION AND GENERAL TOOLS}

In this section we give some notation and note some general properties of $\varrho$ variation and $\lambda$-jumps. The properties will be used later in the paper.

Let $\varrho \geq 1$. For a sequence of numbers $x=\left(x_{n}\right)$, we use the notation $V_{\varrho}(x)=$ $V_{\varrho}\left(\left(x_{n}\right)\right)$ to denote the $\varrho$-variation number defined by the following:

$$
V_{\varrho}\left(\left(x_{n}\right)\right)=\sup _{\left(n_{j}\right)}\left(\sum_{k=1}^{\infty}\left|x_{n_{j}}-x_{n_{j+1}}\right|^{\varrho}\right)^{\frac{1}{\varrho}},
$$

where the supremum is taken over all increasing sequences $\left(n_{j}\right)$. When the sequence $x=\left(x_{n}\right)$ is clear from the context, we use $V_{\varrho}(x)$ or $V_{\varrho}\left(x_{n}\right)$ to denote $V_{\varrho}\left(\left(x_{n}\right)\right)$.

Similarly, if $T=\left(T_{n}\right)$ denotes a sequence of operators, we will use the notation $V_{\varrho}\left(\left(T_{n} f\right)\right)(x)$ to denote the $\varrho$-variation operator defined by the sequence $\left(T_{n}\right)$ at the point $x$. That is,

$$
V_{\varrho}\left(\left(T_{n} f\right)\right)(x)=\sup _{\left(n_{j}\right)}\left(\sum_{k=1}^{\infty}\left|T_{n_{j}} f(x)-T_{n_{j+1}} f(x)\right|^{\varrho}\right)^{\frac{1}{\varrho}},
$$

where the supremum is taken over all increasing sequences $\left(n_{j}\right)$. Again, when $T=\left(T_{n}\right)$ is clear from the context, we use $V_{\varrho}\left(T_{n} f\right)(x)$ or simply $V_{\varrho}(T f)(x)$ to denote $V_{\varrho}\left(\left(T_{n} f\right)\right)(x)$. Note that $V_{\varrho}\left(T_{n} f\right)(x)=V_{\varrho}\left(T_{n} f(x)\right)$.

We note some important properties of the variation operator. If $\varrho \geq 1$, and $T=\left(T_{n}\right), S=\left(S_{n}\right)$ are two sequences of operators, then

$$
V_{\varrho}((T+S) f)(x) \leq V_{\varrho}(T f)(x)+V_{\varrho}(S f)(x) .
$$

Because for any sequence $\left(a_{n}\right),\left\|a_{n}\right\|_{\ell^{\varrho_{1}}} \leq\left\|a_{n}\right\|_{\ell_{2}}$ when $\varrho_{1} \geq \varrho_{2} \geq 1$, we have

$$
V_{\varrho_{1}}(T f)(x) \leq V_{\varrho_{2}}(T f)(x)
$$

when $\varrho_{1} \geq \varrho_{2}$. Finally, since $(a+b)^{2} \leq 2 a^{2}+2 b^{2}$, we also have

$$
V_{2}(T f)(x) \leq 2 \sqrt{2}\left(\sum_{n}\left(T_{n} f\right)^{2}(x)\right)^{1 / 2} .
$$

Obviously, similar properties apply to variation numbers for sequences.

During the proofs of the main results we will need to work with a family of conditional expectation operators. Let $\mathcal{F}_{n}$ denote the $n$th dyadic $\sigma$-field associated with $\mathbb{T}$. That is, $\mathcal{F}_{0}$ consists of the two sets, $\{\emptyset,[-\pi, \pi)\}$, and $\mathcal{F}_{i+1}$ is obtained 
from $\mathcal{F}_{i}$ by dividing each atom of $\mathcal{F}_{i}$ into two disjoint intervals of equal length. Throughout the paper, let $E_{n} f=E\left(f \mid \mathcal{F}_{n}\right)$, where integration is with respect to normalized Lebesgue measure on $\mathbb{T}$.

Remark 2.1. To see that variation inequalities are not a consequence of convergence, we note the following simple example of a sequence of operators that converge to zero a.e., but which fail to satisfy a variation inequality for every $\varrho>0$. On $[0,1)$, let $T_{2 n} f(x)=\frac{1}{\ln 2 n} f(x)$, and let $T_{2 n+1} f(x)=\frac{-1}{\ln 2 n} f(x)$. Then for any $f$, we have $V_{\varrho}\left(T_{n} f\right)(x)=\infty$ on $\{x: f(x) \neq 0\}$.

Definition 2.2. Let $x=\left(x_{n}\right)$ denote a sequence of real numbers. We define

$$
\begin{array}{r}
\Lambda(x, \lambda)=\Lambda\left(\left(x_{n}\right), \lambda\right)=\sup \left\{n: \text { there exists } s_{1}<t_{1} \leq s_{2}<t_{2} \cdots \leq s_{n}<t_{n}\right. \\
\text { such that } \left.\left|x_{t_{i}}-x_{s_{i}}\right| \geq \lambda \text { for } i=1,2, \ldots, n\right\} .
\end{array}
$$

When the sequence $x=\left(x_{n}\right)$ is clear from the context, we use $\Lambda(x, \lambda)$ or $\Lambda\left(x_{n}, \lambda\right)$ to denote $\Lambda\left(\left(x_{n}\right), \lambda\right)$.

The operator $\Lambda(x, \lambda)$ gives the number of times the sequence $x=\left(x_{n}\right)$ changes by a distance $\lambda$. For a continuously indexed system, $x=\left(x_{t}\right)_{t \in \mathbb{R}}, \Lambda(x, \lambda)$ is defined in the analogous way.

Remark 2.3. We first show there is a triangle like inequality for the jump numbers. Let $x=\left(x_{n}\right)$ and $y=\left(y_{n}\right)$ be two sequences. Note

$$
\begin{array}{r}
\Lambda(x+y, \lambda)=\sup \left\{n: \text { there is } s_{1}<t_{1} \leq s_{2}<t_{2} \cdots \leq s_{n}<t_{n}\right. \text { such that } \\
\\
\left.\quad\left|x_{t_{i}}+y_{t_{i}}-x_{s_{i}}-y_{s_{i}}\right|>\lambda\right\}
\end{array}
$$

and

$$
\left|x_{t_{i}}+y_{t_{i}}-x_{s_{i}}-y_{s_{i}}\right| \leq\left|x_{t_{i}}-x_{s_{i}}\right|+\left|y_{t_{i}}-y_{s_{i}}\right| \text {. }
$$

Consequently if $\left|\left(x_{t_{i}}+y_{t_{i}}\right)-\left(y_{s_{i}}+y_{s_{i}}\right)\right|>\lambda$, then at least one of the two terms on the right-hand side must be greater than $\lambda / 2$. From this we see that

$$
\Lambda(x+y, \lambda) \leq \Lambda\left(x, \frac{\lambda}{2}\right)+\Lambda\left(y, \frac{\lambda}{2}\right) .
$$

Next, we relate the jump number $\Lambda(x, \lambda)$ to the $\varrho$-variation number $V_{\varrho}(x)$. Let $s_{1}<t_{1} \leq s_{2}<t_{2} \leq \cdots \leq s_{n}<t_{n}$ be the indices used in the definition of $\Lambda(x, \lambda)$. That is, $\left|x_{t_{j}}-x_{s_{j}}\right|>\lambda$ for $j=1,2, \ldots, n$. From this we see that

$$
\lambda \Lambda(x, \lambda)^{\frac{1}{\varrho}} \leq\left(\sum_{j=1}^{n}\left|x_{t_{j}}-x_{s_{j}}\right|^{\varrho}\right)^{\frac{1}{\varrho}} \leq V_{\varrho}(x) .
$$

Definition 2.4. Let $1 \leq p<\infty$. For two sequences of $L^{p}$ operators $\left(A_{n}\right)$ and $\left(B_{n}\right)$, we say that $\left(A_{n}\right)$ and $\left(B_{n}\right)$ are equivalent, and will write $\left(A_{n}\right) \sim_{p}\left(B_{n}\right)$, if for any sequence $\left(\nu_{n}\right)$ of complex numbers with $\left|\nu_{n}\right| \leq 1$ and for every $f \in L^{p}$, we have

$$
\left\|\sum_{n}\left(A_{n} f-B_{n} f\right) \cdot \nu_{n}\right\|_{p} \leq c\|f\|_{p}
$$

for some constant $c$ independent of $\left(\nu_{n}\right)$ and $f$.

Theorem 2.5. If $\left(A_{n}\right) \sim_{p}\left(B_{n}\right)$, then

$$
\left\|\left(\sum_{n}\left|A_{n} f-B_{n} f\right|^{2}\right)^{1 / 2}\right\|_{p}^{p} \leq c\|f\|_{p}^{p} .
$$


Proof. Let $\nu_{n}=r_{n}$, the $n$th Rademacher function. First use Khinchine's inequality followed by Fubini's theorem. Then we can apply the hypothesis of the theorem. The details are as follows:

$$
\begin{aligned}
\left\|\left(\sum_{n}\left|A_{n} f-B_{n} f\right|^{2}\right)^{1 / 2}\right\|_{p}^{p} & =\frac{1}{2 \pi} \int_{-\pi}^{\pi}\left(\sum_{n}\left|A_{n} f(x)-B_{n} f(x)\right|^{2}\right)^{p / 2} d x \\
& \leq c \int_{-\pi}^{\pi} \int_{0}^{1}\left(\sum_{n}\left(A_{n} f(x)-B_{n} f(x)\right) r_{n}(t)\right)^{p} d t d x \\
& =c \int_{0}^{1} \int_{-\pi}^{\pi}\left(\sum_{n}\left(A_{n} f(x)-B_{n} f(x)\right) r_{n}(t)\right)^{p} d x d t \\
& \leq c \int_{0}^{1}\|f\|_{p}^{p} d t \\
& =c\|f\|_{p}^{p} .
\end{aligned}
$$

Remark 2.6. The above argument shows that the condition

$$
\left\|\left(\sum_{n}\left|A_{n} f-B_{n} f\right|^{2}\right)^{1 / 2}\right\|_{p}^{p} \leq c\|f\|_{p}^{p}
$$

is weaker than $\left(A_{n}\right) \sim_{p}\left(B_{n}\right)$. In fact, all the results in this paper are true under this weaker condition.

In case $p=\infty$, we say $\left(A_{n}\right)$ and $\left(B_{n}\right)$ are equivalent and write $\left(A_{n}\right) \sim_{\infty}\left(B_{n}\right)$ if

$$
\left\|\sum_{n}\left(A_{n} f-B_{n} f\right) \cdot \nu_{n}\right\|_{B M O} \leq c\|f\|_{\infty}
$$

for some constant $c$ independent of $\left(\nu_{n}\right)$ and $f$.

Lemma 2.7. Let $1 \leq p<\infty, \varrho \geq 2$, and $\left(A_{n}\right) \sim_{p}\left(B_{n}\right)$ (or under the weaker condition of (2.4) $)$.

(1) If

$$
\left\|V_{\varrho}(A f)\right\|_{p} \leq c\|f\|_{p}
$$

then

$$
\left\|V_{\varrho}(B f)\right\|_{p} \leq c\|f\|_{p}
$$

(2) If

$$
V_{\varrho}(A f)(x)=\infty \quad \text { a.e. }
$$

for some $f \in L^{\infty}$, then

$$
V_{\varrho}(B f)(x)=\infty \quad \text { a.e. }
$$

(3) If

$$
\left\|\Lambda(A f, \lambda)^{1 / 2}\right\|_{p} \leq \frac{c}{\lambda}\|f\|_{p},
$$

then

$$
\left\|\Lambda(B f, \lambda)^{1 / 2}\right\|_{p} \leq \frac{c}{\lambda}\|f\|_{p} .
$$


Proof. For (11), using (2.1), since

$$
V_{\varrho}((A-B) f)(x) \leq V_{2}((A-B) f)(x) \leq 2 \sqrt{2}\left(\sum_{n}\left|\left(A_{n} f-B_{n} f\right)(x)\right|^{2}\right)^{1 / 2},
$$

the lemma follows from the triangle inequality and Theorem 2.5.

For the proof of (2) note that $V_{\varrho}(A)=V_{\varrho}(A-B+B) \leq V_{\varrho}(A-B)+V_{\varrho}(B) \leq$ $V_{2}(A-B)+V_{\varrho}(B)$. Arguing as above, we have that the first term on the right-hand side is finite a.e. Since the left-hand side is infinite a.e. the second term on the right-hand side must also be infinite a.e.

For (3), by (2.2), we have

$$
\Lambda(B f, \lambda)(x) \leq \Lambda\left((B-A) f, \frac{\lambda}{2}\right)(x)+\Lambda\left(A f, \frac{\lambda}{2}\right)(x) .
$$

Taking the square root of both sides, and using the fact that $\sqrt{a+b} \leq \sqrt{a}+\sqrt{b}$, we see that

$$
\lambda \sqrt{\Lambda(B f, \lambda)(x)} \leq \lambda \sqrt{\Lambda\left((B-A) f, \frac{\lambda}{2}\right)(x)}+\lambda \sqrt{\Lambda\left(A f, \frac{\lambda}{2}\right)(x)} .
$$

Taking the $p$ th norm of both sides, we see that

$$
\|\lambda \sqrt{\Lambda(B f, \lambda)}\|_{p} \leq\left\|\lambda \sqrt{\Lambda\left((B-A) f, \frac{\lambda}{2}\right)}\right\|_{p}+\left\|\lambda \sqrt{\Lambda\left(A f, \frac{\lambda}{2}\right)}\right\|_{p} .
$$

Now the result follows from the assumption and equations (2.3) and (2.4).

Throughout the paper we will make use of known results about an additional family of operators, in particular, the family of Lebesgue differentiation operators.

Let

$$
D_{n} f(x)=\frac{n}{2 \pi} \int_{\frac{-\pi}{n}}^{\frac{\pi}{n}} f(x+t) d t=\frac{1}{2 \pi} \int_{-\pi}^{\pi} n \chi_{\left[-\frac{\pi}{n}, \frac{\pi}{n}\right]}(t) f(x-t) d t .
$$

The reason to introduce the family of operators, $\left(D_{n}\right)$, is that we already know a variation inequality for these operators (see [2]), and these operators can be used to approximate the behavior of other families of operators. This approximation is the content of the following lemma.

Lemma 2.8. Suppose a sequence $\left(A_{n}\right)$ of kernels satisfies

$$
\begin{aligned}
\left|1-\widehat{A}_{n}(j)\right| & \leq c \cdot|j / n|^{\sigma}, & & |j| \leq n, \\
\left|\widehat{A}_{n}(j)\right| & \leq c \cdot|n / j|^{\sigma}, & & |j|>n,
\end{aligned}
$$

for some $\sigma>0$. Then for any lacunary sequence $\left(n_{k}\right)$ we have $\left(A_{n_{k}}\right) \sim_{2}\left(D_{n_{k}}\right)$.

Proof. We need to show for any sequence $\left(\nu_{k}\right)$ of complex numbers with $\left|\nu_{k}\right| \leq 1$ and for any $f \in L^{2}$,

$$
\left\|\sum_{k}\left(A_{n_{k}} f-D_{n_{k}} f\right) \cdot \nu_{k}\right\|_{2} \leq c\|f\|_{2}
$$

for some positive constant $c>0$ independent of $\left(\nu_{k}\right)$ and $f$. 
Since

$$
\begin{aligned}
\left\|\sum_{k}\left(A_{n_{k}} f-D_{n_{k}} f\right) \cdot \nu_{k}\right\|_{2}^{2} & =\frac{1}{2 \pi} \int_{-\pi}^{\pi} \sum_{k=1}^{\infty}\left|\left(\left(A_{n_{k}} f\right)(x)-\left(D_{n_{k}} f\right)(x)\right) \cdot \nu_{k}\right|^{2} d x \\
& =\sum_{j}\left|\sum_{n=1}^{\infty}\left(\widehat{A}_{n_{k}}(j) \widehat{f}(j)-\widehat{D}_{n_{k}}(j) \widehat{f}(j)\right) \nu_{k}\right|^{2} \\
& =\sum_{j}|\widehat{f}(j)|^{2}\left|\sum_{k=1}^{\infty}\left(\widehat{A}_{n_{k}}(j)-\widehat{D}_{n_{k}}(j)\right) \nu_{k}\right|^{2} \\
& \leq \sum_{j}|\widehat{f}(j)|^{2}\left|\sum_{k=1}^{\infty}\right| \widehat{A}_{n_{k}}(j)-\left.\widehat{D}_{n_{k}}(j)\right|^{2}
\end{aligned}
$$

it is enough to show that

$$
\sum_{k=1}^{\infty}\left|\widehat{A}_{n_{k}}(j)-\widehat{D}_{n_{k}}(j)\right|
$$

is bounded uniformly in $j$.

Computing the Fourier series, we see that

$$
\widehat{D}_{n_{k}}(j)=n_{k} \frac{1}{2 \pi} \int_{\frac{-\pi}{n_{k}}}^{\frac{\pi}{n_{k}}} e^{i j t} d t=n_{k} \frac{\sin \left(\pi j / n_{k}\right)}{\pi j} .
$$

Expanding

$$
\sin \left(\pi j / n_{k}\right)=\pi j / n_{k}+O\left(\pi j / n_{k}\right)^{3},
$$

we see that for $k$ such that $|j| \leq n_{k}$, we have

$$
\left|\widehat{A}_{n_{k}}(j)-\widehat{D}_{n_{k}}(j)\right| \leq\left|\widehat{A}_{n_{k}}(j)-1\right|+\left|\widehat{D}_{n_{k}}(j)-1\right| \leq C\left(\frac{j}{n_{k}}\right)^{\sigma_{1}}
$$

where $\sigma_{1}=\min \{2, \sigma\}$.

Similarly, for $k$ such that $|j|>n_{k}$, we have

$$
\left|\widehat{A}_{n_{k}}(j)-\widehat{D}_{n_{k}}(j)\right| \leq\left|\widehat{A}_{n_{k}}(j)\right|+\left|\widehat{D}_{n_{k}}(j)\right| \leq C\left(\frac{n_{k}}{j}\right)^{\sigma_{2}},
$$

where $\sigma_{2}=\min \{1, \sigma\}$.

Therefore, by the above two inequalities,

$$
\begin{aligned}
\sum_{k=1}^{\infty}\left|\widehat{A}_{n_{k}}(j)-\widehat{D}_{n_{k}}(j)\right| & =\sum_{\left\{k: n_{k}<|j|\right\}}\left|\widehat{A}_{n_{k}}(j)-\widehat{D}_{n_{k}}(j)\right| \\
& +\sum_{\left\{k: n_{k} \geq|j|\right\}}\left|\widehat{D}_{n_{k}}(j)-\widehat{K}_{n_{k}}(j)\right| \\
& \leq \sum_{\left\{k: n_{k}<|j|\right\}} C\left(\frac{n_{k}}{j}\right)^{\sigma_{2}}+\sum_{\left\{k: n_{k} \geq|j|\right\}} C\left(\frac{j}{n_{k}}\right)^{\sigma_{1}} \\
& \leq C .
\end{aligned}
$$

This completes the proof.

Remark 2.9. If $E_{n}$ denotes the conditional expectation operator with respect to the dyadic $\sigma$-field with $2^{n}$ atoms, then in [3] it was shown that $\left(E_{n}\right) \sim_{p}\left(D_{2^{n}}\right)$ for $1<p<\infty$. Since $\sim_{p}$ is an equivalence relation, this will allow us to transfer properties of the conditional expectation operator to other families of operators. 
The idea of comparing $D_{2^{n}}$ and $E_{n}$ was used in [2, but there the comparison was via a square function rather than the above equivalence relation.

\section{3. $L^{2}$ RESUlts FOR THE FEJÉR KERNEL}

To prove the $L^{2}$ variation result for the Fejér kernel we need to split the variation into two parts. One part will involve the case when there are long gaps between $n_{k}$ and $n_{k+1}$, and the other part will involve shorter gaps. It turns out that the longer gaps are the more difficult to deal with, and are the source of the requirement that $\varrho>2$. We will see that the short gaps are controlled by an operator that has been studied as early as 1925 by Kaczmarz [4] and 1926 by Zygmund [9]. To study the case of "long gaps" we will approximate the operators of interest by simpler operators that are already understood.

3.1. The long variation. In this section we will study the variation along lacunary sequences. In particular, we will prove the following theorem.

Theorem 3.1. Let $\left(n_{k}\right)$ be a lacunary sequence. Then $\left(\sigma_{n_{k}}\right) \sim_{2}\left(D_{n_{k}}\right)$. Further, for $\varrho>2$ we have $\left\|V_{\varrho}\left(\sigma_{n_{k}} f\right)\right\|_{2} \leq c\left(\varrho,\left(n_{k}\right)\right)\|f\|_{2}$.

Remark 3.2. We will see later that the constant in the above inequality does not actually depend on the sequence $\left(n_{k}\right)$, but that will not be established until later in the paper.

Proof. The Fejér kernel has a Fourier transform given by

$$
\widehat{K}_{n}(j)= \begin{cases}\left(1-\frac{|j|}{n+1}\right) & \text { if }|j| \leq n, \\ 0 & \text { if }|j|>n .\end{cases}
$$

Applying Lemma 2.8 we see that $\left(\sigma_{n_{k}}\right) \sim_{2}\left(D_{n_{k}}\right)$. From [2] we know that for $\varrho>2$ we have $\left\|V_{\varrho}\left(D_{n_{k}} f\right)\right\|_{2} \leq c(\varrho)\|f\|_{2}$. Now apply Lemma 2.7 .

Remark 3.3. In the special case when $\left(n_{k}\right)=\left(2^{k}\right)$ we will sometimes refer to $V_{\varrho}\left(\sigma_{2^{n}} f\right)$ as the long variation. One reason to work with this fixed lacunary sequence is to have a fixed constant for later computation.

Theorem 3.4. Let $S_{n} f(x)$ be the nth (symmetric) partial sum of the Fourier series for $f$. That is,

$$
S_{n} f(x)=\sum_{j=-n}^{n} \hat{f}(j) e^{i j x} .
$$

If $\left(n_{k}\right)$ is any lacunary sequence then $\left(S_{n_{k}}\right) \sim_{2}\left(D_{n_{k}}\right),\left(S_{n_{k}}\right) \sim_{2}\left(\sigma_{n_{k}}\right)$ and for $\varrho>2$ we have $\left\|V_{\varrho}\left(S_{n_{k}} f\right)\right\|_{2} \leq c\left(\varrho,\left(n_{k}\right)\right)\|f\|_{2}$.

Proof. Note that

$$
\widehat{S}_{n}(j)= \begin{cases}1, & |j| \leq n, \\ 0, & |j|>n .\end{cases}
$$

Since the hypothesis of Lemma 2.8 is satisfied, we have $\left(S_{n_{k}}\right) \sim_{2}\left(D_{n_{k}}\right)$ and applying Theorem 3.1, $\left(S_{n_{k}}\right) \sim_{2}\left(\sigma_{n_{k}}\right)$. Now apply Lemma 2.7] as above. 
An immediate corollary of Theorem 3.4 and Remark 2.9 is the following.

Corollary 3.5. With $S_{n}$ as above, we have $\left(S_{2^{n}}\right) \sim_{2}\left(E_{n}\right)$ and

$$
\left(\sum_{n=1}^{\infty}\left|S_{2^{n}} f(x)-E_{n} f(x)\right|^{2}\right)^{\frac{1}{2}}
$$

is a bounded operator on $L^{2}$.

3.2. The $L^{2}$ short variation. We now consider the short variation. For each integer $k>0$, let $I_{k}=\left[2^{k}, 2^{k+1}\right)$. Define the "short variation operator" by

$$
V_{\varrho}^{S}(K f)(x)=\left(\sum_{k} \sup _{\left(n_{j}\right) \nearrow} \sum_{\left\{j:\left[n_{j}, n_{j+1}\right) \subset I_{k}\right\}}\left|\sigma_{n_{j}} f(x)-\sigma_{n_{j+1}} f(x)\right|^{\varrho}\right)^{\frac{1}{\varrho}} .
$$

Note that the sequence $\left(n_{j}\right)$ can, and usually does, depend on $x$.

Since when $\varrho \geq 2$,

$$
V_{\varrho}^{S}(K f)(x) \leq V_{2}^{S}(K f)(x),
$$

we will study $V_{2}^{S}(K f)$ for the rest of the section.

In the study of this operator we will encounter the operator

$$
G f(x)=\left(\sum_{\ell=1}^{\infty} \ell\left|\sigma_{\ell} f(x)-\sigma_{\ell-1} f(x)\right|^{2}\right)^{\frac{1}{2}} .
$$

This is the operator studied by Zygmund. See [10], Chapter 15, page 223.

Remark 3.6. In Zygmund 10] this operator is denoted by $\gamma_{2}(x, f)$ and at first glance appears different from $G f(x)$. However, easy algebra shows that they are the same. Zygmund first considers the power series case, but later in the chapter shows how to deduce the more general case, needed here, from the power series case. Stein [7] mentions $G f$ explicitly.

Theorem 3.7. The operator $V_{2}^{S}(K f)(x)$ satisfies $\left\|V_{2}^{S}(K f)\right\|_{2} \leq c\|f\|_{2}$.

Proof. Let $S_{k} f(x)$ denote the sum over the $k$ th dyadic block $I_{k}=\left[2^{k}, 2^{k+1}\right)$, that is, let

$$
S_{k} f(x)=\left(\sup _{\left(n_{j}\right) \nearrow} \sum_{\left\{j:\left[n_{j}, n_{j+1}\right) \subset I_{k}\right\}}\left|\sigma_{n_{j}} f(x)-\sigma_{n_{j+1}} f(x)\right|^{2}\right)^{\frac{1}{2}}
$$

Then

$$
V_{2}^{S}(K f)(x)=\left(\sum_{k=1}^{\infty}\left|S_{k} f(x)\right|^{2}\right)^{\frac{1}{2}}
$$

Using the fact that

$$
\sigma_{n_{j}} f(x)-\sigma_{n_{j+1}} f(x)=\sum_{\ell=n_{j}}^{n_{j+1}-1} \sigma_{\ell} f(x)-\sigma_{\ell+1} f(x)
$$


and the fact that $2^{k} \leq \ell<2^{k+1}$ when $\ell \in I_{k}$, we can dominate $S_{k} f(x)$ as follows:

$$
\begin{aligned}
S_{k} f(x) & =\left(\sup _{\left(n_{j}\right) \nearrow} \sum_{\left\{j:\left[n_{j}, n_{j+1}\right) \subset I_{k}\right\}}\left|\sigma_{n_{j}} f(x)-\sigma_{n_{j+1}} f(x)\right|^{2}\right)^{\frac{1}{2}} \\
& \leq\left(\sup _{\left(n_{j}\right) \nearrow} \sum_{\left\{j:\left[n_{j}, n_{j+1}\right) \subset I_{k}\right\}}\left|\sum_{\ell=n_{j}}^{n_{j+1}-1}\left(\sigma_{\ell} f(x)-\sigma_{\ell+1} f(x)\right)\right|^{2}\right)^{\frac{1}{2}} \\
& \leq \sup _{\left(n_{j}\right) \nearrow} \sum_{\left\{j:\left[n_{j}, n_{j+1}\right) \subset I_{k}\right\}}\left|\sum_{\ell=n_{j}}^{n_{j+1}-1}\left(\sigma_{\ell} f(x)-\sigma_{\ell+1} f(x)\right)\right| \\
& \leq \sup _{\left(n_{j}\right) \nearrow} \sum_{\left\{j:\left[n_{j}, n_{j+1}\right) \subset I_{k}\right\}} \sum_{\ell=n_{j}}^{n_{j+1}-1}\left|\sigma_{\ell} f(x)-\sigma_{\ell+1} f(x)\right| \\
& \leq \sum_{\ell=2^{k}}^{2^{k+1}-1}\left|\sigma_{\ell} f(x)-\sigma_{\ell+1} f(x)\right| \\
& \leq \sum_{\ell=2^{k}}^{2^{k+1}-1} \frac{1}{\sqrt{\ell+1}} \sqrt{\ell+1}\left|\sigma_{\ell} f(x)-\sigma_{\ell+1} f(x)\right| \\
& \leq\left(\sum_{\ell=2^{k}}^{2^{k+1}-1} \frac{1}{\ell+1}\right)^{\frac{1}{2}}\left(\sum_{\ell=2^{k}}^{2^{k+1}-1}(\ell+1)\left|\sigma_{\ell} f(x)-\sigma_{\ell+1} f(x)\right|^{2}\right)^{\frac{1}{2}} \\
& \leq C\left(\sum_{\ell=2^{k}}^{2^{k+1}-1}(\ell+1)\left|\sigma_{\ell} f(x)-\sigma_{\ell+1} f(x)\right|^{2}\right)^{\frac{1}{2}} .
\end{aligned}
$$

Hence

$$
V_{2}^{S}(K f)(x)=\left(\sum_{k=1}^{\infty}\left|S_{k} f(x)\right|^{2}\right)^{\frac{1}{2}} \leq C G f(x) .
$$

Thus we will be done if we can show that $\|G f\|_{2} \leq c\|f\|_{2}$, but as remarked above, this is contained in [10].

We can now complete the proof that the operator $V_{\varrho}\left(\sigma_{n} f\right)$ is bounded on $L^{2}$. For an increasing sequence $\left\{n_{j}\right\}$ and $j \geq 1$, let $u_{j}=\max \left\{k: n_{j}+1 \leq 2^{k} \leq n_{j+1}\right\}$ and $v_{j}=\min \left\{k: n_{j}+1 \leq 2^{k} \leq n_{j+1}\right\}$ if the set is not empty. Otherwise, they are undefined. Then either the term

$$
\left|\sigma_{n_{j+1}} f-\sigma_{n_{j}+1} f\right|^{\varrho}
$$

is part of the short variation when the set $\left\{k: n_{j}+1 \leq 2^{k} \leq n_{j+1}\right\}$ is empty, or

$\left|\sigma_{n_{j+1}} f-\sigma_{n_{j}+1} f\right|^{\varrho} \leq 3^{\varrho}\left(\left|\sigma_{n_{j+1}} f-\sigma_{2^{u_{j}}} f\right|^{\varrho}+\left|\sigma_{2^{u_{j}}} f-\sigma_{2^{v_{j}}} f\right|^{\varrho}+\left|\sigma_{2^{v_{j}}} f-\sigma_{n_{j}+1} f\right|^{\varrho}\right)$

when the set is not empty. In the latter case, the first and the third terms are part of the short variation operator and the middle term is part of the long variation or zero. So the variation is bounded by at most three times the sum of the short and long variations. Both of these are bounded in $L^{2}$, so the proof of Theorem [1.5 in the case $p=2$ is complete. 


\section{The CASe $2 \leq p<\infty$}

In this section we maintain the notation from the previous section. We again consider the case of long variation and short variation separately. We will show that the "long variation" operator will map $L^{\infty}$ to dyadic-BMO, and hence we can interpolate between $L^{2}$ and dyadic-BMO, obtaining $L^{p}$ results in the case of the long variation operator for $2 \leq p<\infty$. To study the short variation we can again dominate the short variation by the operator

$$
G f(x)=\left(\sum_{\ell=1}^{\infty} \ell\left|\sigma_{\ell} f(x)-\sigma_{\ell-1} f(x)\right|^{2}\right)^{\frac{1}{2}},
$$

which Zygmund [10] showed to be bounded for all $p, 1<p<\infty$.

We will first prove the following two theorems.

Theorem 4.1. The long variation operator $V_{\varrho}\left(\sigma_{2^{n}} f\right)$ is a bounded operator from $L^{\infty}$ to dyadic-BMO.

Theorem 4.2. Let $\left(n_{k}\right)$ be a lacunary sequence; then $\left(\sigma_{n_{k}}\right) \sim_{\infty}\left(D_{n_{k}}\right)$.

To prove these theorems we will use the following result from [2] (suitably restated for our purposes here).

Theorem 4.3. Let $A$ be an operator defined on $L^{\infty}(\mathbb{T})$. Assume the following five properties:

(1) The operator $A$ is a bounded $L^{2} \rightarrow L^{2}$ operator.

(2) There is an operator $B$ which satisfies $|A f(x)-A f(y)| \leq C \cdot B f(x, y)$ for some constant $C$ and every $f, x, y$, and which satisfies the following three properties.

(3) With some constant $C$, if $f=g+h$, then

$$
B f(x, y) \leq C \cdot(B g(x, y)+B h(x, y)) .
$$

(4) With some constant $C$, we have

$$
B f(x, y) \leq C \cdot \max \{A f(x), A f(y)\} .
$$

(5) There is a constant $C$ so that for any interval $I \subset \mathbb{T}$, if $\tilde{I}$ denotes an interval with the same center as $I$, but three times the length, and $f \in L^{\infty}$ is supported on $\mathbb{T} \backslash \tilde{I}$, we have

$$
\sup _{x, y \in I} B f(x, y) \leq C \cdot\|f\|_{L^{\infty}} .
$$

Then there is a constant $C$ such that $\|A f\|_{B M O} \leq C\|f\|_{\infty}$. The constant $C$ depends only on the constants in (2), (3), (4), (5) and the $L^{2} \rightarrow L^{2}$ bound of $A$.

Remark 4.4. A similar result is true if we restrict the intervals $I$ in (5) to dyadic intervals, but then the conclusion is that $A$ maps $L^{\infty}$ to dyadic- $B M O$. This is the case that we will use, since it is sufficient for our needs.

Condition (5) of Theorem 4.3 usually needs most of the work to verify when applying this theorem. We formulate the following lemma for verifying condition (5) in our setting. 
Lemma 4.5. Let $\left(T_{n}\right)$ be a family of operators on $L^{\infty}(\mathbb{T})$ given by

$$
T_{n} f(x)=\left(W_{n} * f\right)(x)=\frac{1}{2 \pi} \int_{-\pi}^{\pi} W_{n}(x-t) f(t) d t .
$$

If there exists a constant $c>0$ such that for any $x, y \in \mathbb{T}$,

$$
\left|W_{n}(x)\right| \leq \frac{c}{n x^{2}}
$$

and

$$
\left|W_{n}(x)-W_{n}(y)\right| \leq \begin{cases}c n^{2}|x-y|, & \max \{n|x-y|, \min \{n|x|, n|y|\}\}<1, \\ c\left(\frac{1}{x^{2}}+\frac{1}{y^{2}}\right)|x-y|, & \min \{n|x|, n|y|\} \geq 1,\end{cases}
$$

then there exists a constant $C>0$ such that for $f \in L^{\infty}$ with support outside $\tilde{I}$, and $x, y \in I$,

$$
\left|T_{n} f(x)-T_{n} f(y)\right| \leq \begin{cases}C n|I|\|f\|_{\infty}, & n|I|<1 \\ C(n|I|)^{-1}\|f\|_{\infty}, & n|I| \geq 1\end{cases}
$$

Further, for any lacunary sequence $\left(n_{k}\right)$ and any $\varrho \geq 1$ the operator

$$
H_{\varrho} f(x, y)=\left(\sum_{k=1}^{\infty}\left|T_{n_{k}} f(x)-T_{n_{k}} f(y)\right|^{\varrho}\right)^{\frac{1}{\varrho}}
$$

satisfies

$$
\sup _{x, y \in I} H_{\varrho} f(x, y) \leq C \cdot\|f\|_{\infty} .
$$

Proof. For notational convenience we will only give the proof in the case $\left(n_{k}\right)=$ $\left(2^{k}\right)$. The general case follows in exactly the same way. We show the second half of the lemma first. Suppose (4.3) holds. Let $I$ be any interval in $\mathbb{T}$ and $f \in L^{\infty}$ be supported on $\mathbb{T} \backslash \tilde{I}$. We need to show

$$
\sup _{x, y \in I} H_{\varrho} f(x, y) \leq C\|f\|_{\infty}
$$

for a constant $C$ independent of $I$ and $f$.

Note that

$$
\begin{aligned}
& \sum_{k}\left|T_{2^{n_{k}}} f(x)-T_{2^{n_{k}}} f(y)\right|^{\varrho} \\
& \quad \leq \sum_{2^{n_{k}<1 /|I|}}\left|T_{2^{n_{k}}} f(x)-T_{2^{n_{k}}} f(y)\right|^{\varrho}+\sum_{2^{n_{k}} \geq 1 /|I|}\left|T_{2^{n_{k}}} f(x)-T_{2^{n_{k}}} f(y)\right|^{\varrho} \\
& \quad=A+B .
\end{aligned}
$$

For $A$, since $2^{n_{k}}|I|<1$, by (4.3), each term in the sum defining $A$ satisfies

$$
\left|T_{2^{n_{k}}} f(x)-T_{2^{n_{k}}} f(y)\right| \leq C 2^{n_{k}}|I|\|f\|_{\infty} .
$$

Thus, summing the geometric series, we have

$$
A \leq \sum_{\left\{n_{k}: 2^{n_{k}}<1 /|I|\right\}} C\left(2^{n_{k}}|I|\|f\|_{\infty}\right)^{\varrho} \leq C\|f\|_{\infty}^{\varrho} .
$$

$B$ is shown similarly. Consequently in only remains to show (4.3). 
Assume $n|I|<1$. Let $x, y \in I$. Since $f$ has support outside $\tilde{I}$, we see for $t$ in the support of $f, \min \{|x-t|,|y-t|\}>|I|$. Let

$$
R(x, y, n)=\left\{t: \min \{|x-t|,|y-t|\}>|I|, \min \{|x-t|,|y-t|\}<\frac{1}{n}\right\},
$$

and

$$
S(x, y, n)=\left\{t: \min \{|x-t|,|y-t|\}>|I|, \min \{|x-t|,|y-t|\} \geq \frac{1}{n}\right\} .
$$

We have the estimate

$$
\begin{aligned}
\mid T_{n} f(x)- & T_{n} f(y)|=| \int_{-\pi}^{\pi}\left(W_{n}(x-t)-W_{n}(y-t)\right) f(t) d t \mid \\
\leq & \left|\int_{R(x, y, n)}\left(W_{n}(x-t)-W_{n}(y-t)\right) f(t) d t\right| \\
& +\left|\int_{S(x, y, n)}\left(W_{n}(x-t)-W_{n}(y-t)\right) f(t) d t\right| \\
= & A(n)+B(n) .
\end{aligned}
$$

We first estimate $A(n)$. Since $\min \{|x-t|,|y-t|\}<\frac{1}{n}$ and $|x-y| \leq|I|<\frac{1}{n}$, we have $\max \{|x-t|,|y-t|\} \leq|x-y|+\min \{|x-t|,|y-t|\}<2 / n$. Thus $R(x, y, n) \subset$ $\{|x-t|<2 / n\} \cup\{|y-t|<2 / n\}$. Because $x, y \in I$ and $|x-y| \leq|I|<1 / n$, we have $\max \{n|x-y|, \min \{n|x-t|, n|y-t|\}\}<1$. So by (4.2)

$$
\left|W_{n}(x-t)-W_{n}(y-t)\right| \leq c n^{2}|x-y| .
$$

Thus,

$$
\begin{aligned}
A(n) & \leq \int_{|I| \leq|x-t| \leq \frac{2}{n}} c n^{2}|x-y||f(t)| d t+\int_{|I| \leq|y-t| \leq \frac{2}{n}} c n^{2}|x-y||f(t)| d t \\
& \leq c n^{2}|I|\|f\|_{\infty} \frac{2}{n} \\
& \leq c n|I|\|f\|_{\infty} .
\end{aligned}
$$

Next, we estimate $B_{n}$. Note in this case, by (4.2),

$$
\begin{aligned}
B_{n} & \leq \int_{S(x, y, n)} \frac{c}{|x-t|^{2}}|x-y||f(t)| d t+\int_{S(x, y, n)} \frac{c}{|y-t|^{2}}|x-y||f(t)| d t \\
& \leq \int_{|x-t| \geq 1 / n} \frac{c}{|x-t|^{2}}|I||f(t)| d t+\int_{|y-t| \geq 1 / n} \frac{c}{|y-t|^{2}}|I||f(t)| d t \\
& \leq c n|I|\|f\|_{\infty} .
\end{aligned}
$$

This finishes the proof of (4.3) in the case $n|I|<1$. Finally, assume $n|I| \geq 1$. By (4.1), we have

$$
\left|W_{n}(x)-W_{n}(y)\right| \leq \frac{c}{n x^{2}}+\frac{c}{n y^{2}} .
$$


So

$$
\begin{aligned}
\left|T_{n} f(x)-T_{n} f(y)\right| & \leq c \int_{\{|x-t|>|I|,|y-t|>|I|\}}\left(\frac{1}{n(x-t)^{2}}+\frac{1}{n(y-t)^{2}}\right)|f(t)| d t \\
& \leq c\left(\int_{|x-t|>|I|} \frac{1}{n(x-t)^{2}}|f(t)| d t+\int_{|y-t|>|I|} \frac{1}{n(y-t)^{2}}|f(t)| d t\right) \\
& \leq \frac{c}{n|I|}\|f\|_{\infty} .
\end{aligned}
$$

This completes the proof.

Proof of Theorem 4.1. We will apply Theorem 4.3, Let

$$
A f(x)=V_{\varrho}\left(\sigma_{2^{n}} f\right)(x) .
$$

Because of Theorem 3.1, we have already established (11) of Theorem 4.3.

We have for any pair $(x, y)$ that

$$
\begin{aligned}
V_{\varrho}\left(\sigma_{2^{n}} f\right)(x)=\sup _{n_{k}}\left(\sum_{k=1}^{\infty}\left|\sigma_{2^{n_{k}}} f(x)-\sigma_{2^{n_{k+1}}} f(x)\right|^{\varrho}\right)^{\frac{1}{\varrho}} \\
\leq \sup _{n_{k}}\left(\sum_{k=1}^{\infty}\left|\left(\sigma_{2^{n_{k}}} f(x)-\sigma_{2^{n_{k}}} f(y)\right)-\left(\sigma_{2^{n_{k+1}}} f(x)-\sigma_{2^{n_{k+1}}} f(y)\right)\right|^{\varrho}\right)^{\frac{1}{\varrho}} \\
\quad+\sup _{n_{k}}\left(\sum_{k=1}^{\infty}\left|\sigma_{2^{n_{k}}} f(y)-\sigma_{2^{n_{k+1}}} f(y)\right|^{\varrho}\right)^{\frac{1}{\varrho}} \\
\leq 2 \sup _{n_{k}}\left(\sum_{k=1}^{\infty}\left|\sigma_{2^{n_{k}}} f(x)-\sigma_{2^{n_{k}}} f(y)\right|^{\varrho}\right)^{\frac{1}{\varrho}}+V_{\varrho}\left(\sigma_{2^{n}} f\right)(y) .
\end{aligned}
$$

Hence, since we can interchange the role of $x$ and $y$, we have

$$
\left|V_{\varrho}\left(\sigma_{2^{n}} f\right)(x)-V_{\varrho}\left(\sigma_{2^{n}} f\right)(y)\right| \leq 2 \sup _{n_{k}}\left(\sum_{k=1}^{\infty}\left|\sigma_{2^{n_{k}}} f(x)-\sigma_{2^{n_{k}}} f(y)\right|^{\varrho}\right)^{\frac{1}{\varrho}} .
$$

We take

$$
B f(x, y)=\left(\sum_{k=1}^{\infty}\left|\sigma_{2^{n_{k}}} f(x)-\sigma_{2^{n_{k}}} f(y)\right|^{\varrho}\right)^{\frac{1}{\varrho}},
$$

where for each pair $(x, y)$ we may use a different sequence $\left(n_{k}\right)$ in the definition. (The sequence is selected in such a way that for each pair $(x, y)$ we get to within a factor of 2 of the supremum.) Properties (2), (3), and (4) follow easily from the triangle inequality for the variation numbers. It remains to check the condition in (5). To this end we will apply Lemma 4.5 by verifying that $K_{n}(x)$ satisfies conditions (4.1) and (4.2) where

$$
\sigma_{n} f(x)=\frac{1}{2 \pi} \int_{-\pi}^{\pi} K_{n}(x-t) f(t) d t
$$

and

$$
K_{n}(x)=\frac{1}{n+1}\left(\frac{\sin \frac{n+1}{2} x}{\sin \frac{x}{2}}\right)^{2} .
$$


Since $\frac{2}{\pi}|x| \leq|\sin x| \leq|x|$ when $|x| \leq \frac{\pi}{2}$, it is easy to see that

$$
\left|K_{n}(x)\right| \leq \frac{c}{n x^{2}}
$$

To verify (4.2), we need only to consider $n \geq 2$. Let $g(x)=\frac{\sin ^{2} m x}{\sin ^{2} x}$, where $m=n+1$. Then

$$
\begin{aligned}
g^{\prime}(x) & =\frac{2 m \sin (m x) \cos (m x) \sin ^{2} x-2 \sin x \cos x \sin ^{2}(m x)}{\sin ^{4} x} \\
& =\frac{2 \sin m x(m \cos m x \sin x-\cos x \sin m x)}{\sin ^{3} x} .
\end{aligned}
$$

Since $\frac{2}{\pi}|x| \leq|\sin x| \leq|x|$, when $|x| \leq \frac{2}{\pi}$, we see that when $0 \leq|n x| \leq 2$, we have $\left|g^{\prime}\left(\frac{x}{2}\right)\right|<c n^{3}$.

Thus, if $\max \{n|x-y|, \min \{n|x|, n|y|\}\}<1$, we have

$$
\max \{n|x|, n|y|\} \leq n|x-y|+\min \{n|x|, n|y|\}<2 .
$$

Using the Mean Value Theorem, this leads to

$$
\left|K_{n}(x)-K_{n}(y)\right| \leq \frac{1}{n}\left|g\left(\frac{x}{2}\right)-g\left(\frac{y}{2}\right)\right| \leq \frac{1}{2 n}\left|g^{\prime}\left(\frac{z}{2}\right)\right||x-y| \leq c n^{2}|x-y|,
$$

where $z$ is between $x$ and $y$, whence $n|z| \leq \max \{n|x|, n|y|\}<2$.

Next, assume $\min \{n|x|, n|y|\} \geq 1$.

$$
\begin{aligned}
\left|K_{n}(x)-K_{n}(y)\right| & \leq \frac{1}{n}\left|\frac{\sin ^{2}\left(\frac{m x}{2}\right)}{\sin ^{2} \frac{x}{2}}-\frac{\sin ^{2}\left(\frac{m y}{2}\right)}{\sin ^{2} \frac{y}{2}}\right| \\
& \leq \frac{1}{n}\left|\frac{\sin ^{2}\left(\frac{m x}{2}\right)}{\sin ^{2} \frac{x}{2}}-\frac{\sin ^{2}\left(\frac{m y}{2}\right)}{\sin ^{2} \frac{x}{2}}\right|+\frac{1}{n}\left|\frac{\sin ^{2}\left(\frac{m y}{2}\right)}{\sin ^{2} \frac{x}{2}}-\frac{\sin ^{2}\left(\frac{m y}{2}\right)}{\sin ^{2} \frac{y}{2}}\right| \\
& \leq \frac{1}{n}\left|\frac{\sin ^{2}\left(\frac{m x}{2}\right)-\sin ^{2}\left(\frac{m y}{2}\right)}{\sin ^{2} \frac{x}{2}}\right|+\frac{1}{n}\left|\frac{1}{\sin ^{2} \frac{x}{2}}-\frac{1}{\sin ^{2} \frac{y}{2}}\right| \\
& =B_{1}(x, y, n)+B_{2}(x, y, n) .
\end{aligned}
$$

Applying the Mean Value Theorem and using the fact that $|\sin x| \geq \frac{2|x|}{\pi}$ when $|x| \leq \frac{\pi}{2}$, we have

$$
\begin{aligned}
B_{1}(x, y, n) & =\frac{1}{n}\left|\frac{\sin ^{2}\left(\frac{m x}{2}\right)-\sin ^{2}\left(\frac{m y}{2}\right)}{\sin ^{2} \frac{x}{2}}\right| \\
& \leq \frac{c}{n} \frac{2 m \frac{|x-y|}{2} \sin (\theta) \cos (\theta)}{x^{2}} \\
& \leq \frac{c|x-y|}{x^{2}},
\end{aligned}
$$

where $\theta$ is between $\frac{m x}{2}$ and $\frac{m y}{2}$. 
For $B_{2}(n)$ we proceed in a similar way. Because $\min \{n|x|, n|y|\} \geq 1$, we have

$$
\begin{aligned}
B_{2}(x, y, n) & =\frac{1}{n}\left|\frac{1}{\sin ^{2} \frac{x}{2}}-\frac{1}{\sin ^{2} \frac{y}{2}}\right| \\
& =\frac{1}{n}\left|\frac{\cos \frac{z}{2}}{\sin ^{3} \frac{z}{2}}\right||x-y| \\
& \leq \frac{c|x-y|}{n|z|^{3}} \\
& \leq \frac{c|x-y|}{n \min \{|x|,|y|\}^{3}} \\
& \leq \frac{c|x-y|}{|x|^{2}}+\frac{c|x-y|}{|y|^{2}} .
\end{aligned}
$$

Consequently,

$$
\left|K_{n}(x)-K_{n}(y)\right| \leq \frac{c|x-y|}{|x|^{2}}+\frac{c|x-y|}{|y|^{2}} .
$$

This completes the proof.

Proof of Theorem 4.2. We have already established the $L^{2}$ case, so it only remains to establish conditions (2), (3), (4) and (5) of Theorem 4.3. We let

$$
B f(x, y)=\sum_{k}\left(\left|\left(\sigma_{n_{k}} f(x)-D_{n_{k}} f(x)\right)-\left(\sigma_{n_{k}} f(y)-D_{n_{k}} f(y)\right)\right| .\right.
$$

This is clearly dominated by

$$
\sum_{k}\left|\sigma_{n_{k}} f(x)-\sigma_{n_{k}} f(y)\right|+\sum_{k}\left|D_{n_{k}} f(x)-D_{n_{k}} f(y)\right|
$$

Conditions (2), (3) and (4) follow easily.

We have already shown that the first term satisfies the required estimates to apply Lemma 4.5. Consequently the first term satisfies condition (55). For the second term, fix an interval $I$. Let $\tilde{I}$ denote an interval with the same center as $I$, but three times the length. We need to estimate

$$
\sum_{k=1}^{\infty}\left|D_{n_{k}} f(x)-D_{n_{k}} f(y)\right|,
$$

where $x \in I, y \in I$ and $f \in L^{\infty}$ is supported on $\mathbb{T} \backslash \tilde{I}$.

First note that if $x \in I$, then $D_{n_{k}} f(x)=\frac{n_{k}}{2 \pi} \int_{x-\pi / n_{k}}^{x+\pi / n_{k}} f(t) d t$ will be zero if $\pi / n_{k}<|I|$ since in this case the domain of integration does not include any of the support of $f$. Hence we only need to sum over those $k$ such that $\pi / n_{k}>|I|$ or $n_{k}<\pi /|I|$. 
Without loss of generality, assume $x<y$. Then

$$
\begin{aligned}
\sum_{n_{k}<\pi /|I|}\left|D_{n_{k}} f(x)-D_{n_{k}} f(y)\right| \\
\quad=\sum_{n_{k}<\pi /|I|}\left|\frac{n_{k}}{2 \pi}\left(\int_{x-\pi / n_{k}}^{x+\pi / n_{k}} f(t) d t-\int_{y-\pi / n_{k}}^{y+\pi / n_{k}} f(t) d t\right)\right| \\
\quad=\sum_{n_{k}<\pi /|I|}\left|\frac{n_{k}}{2 \pi}\left(\int_{x-\pi / n_{k}}^{y-\pi / n_{k}} f(t) d t-\int_{x+\pi / n_{k}}^{y+\pi / n_{k}} f(t) d t\right)\right| \\
\leq \sum_{n_{k}<\pi /|I|} \frac{n_{k}}{2 \pi}\left(\left|\int_{x-\pi / n_{k}}^{y-\pi / n_{k}} f(t) d t\right|+\left|\int_{x+\pi / n_{k}}^{y+\pi / n_{k}} f(t) d t\right|\right) \\
\leq\|f\|_{\infty} \sum_{n_{k}<\pi /|I|} \frac{n_{k}}{\pi}|x-y| \\
\leq\|f\|_{\infty}|I| \sum_{n_{k}<\pi /|I|} \frac{n_{k}}{\pi} \\
\leq\|f\|_{\infty},
\end{aligned}
$$

where in the last line we used the fact that a partial sum of a lacunary series is dominated by a constant times the last term in the sum, and hence

$$
\sum_{n_{k}<\pi /|I|} \frac{n_{k}}{2 \pi} \leq C /|I|
$$

Thus, the second term satisfies condition (5). Hence condition (5) is also satisfied by $B f(x, y)$.

Corollary 4.6. For $2 \leq p<\infty$ we have $\left(\sigma_{n_{k}}\right) \sim_{p}\left(D_{n_{k}}\right)$ and $\left\|V_{\varrho}\left(\sigma_{n_{k}} f\right)\right\|_{p} \leq$ $c\left(p, \varrho,\left(n_{k}\right)\right)\|f\|_{p}$.

Proof. We have already shown that the operators $\sum_{k}\left(\sigma_{n_{k}} f(x)-D_{n_{k}} f(x)\right) \nu_{k}$ are uniformly bounded from $L^{2}$ to $L^{2}$ and from $L^{\infty}$ to BMO. By interpolation, the result follows for all $p, 2 \leq p<\infty$. For the second part of the corollary apply Lemma [2.7] and the fact from [2] that $\left\|V_{\varrho}\left(D_{n_{k}} f\right)\right\|_{p} \leq c(p, \varrho)\|f\|_{p}$.

\section{The CASE $1<p \leq 2$}

In this section, we show that the operator $V_{\varrho}\left(\sigma_{n} f\right)$ is bounded on $L^{p}, 1<p \leq 2$, thus completing the proof of Theorem 1.5 .

Theorem 5.1. Given any lacunary sequence $\left(n_{k}\right)$ we have $\left(\sigma_{n_{k}}\right) \sim_{p}\left(D_{n_{k}}\right)$ for $1<p<\infty$.

Proof. Because $\left(\sigma_{n_{k}}\right) \sim_{p}\left(D_{n_{k}}\right)$ for $2 \leq p<\infty$ we know that the operators

$$
T_{\nu} f(x)=\sum_{k}\left(\sigma_{n_{k}} f(x)-D_{n_{k}} f(x)\right) \nu_{k}
$$

are uniformly bounded from $L^{p}$ to $L^{p}$ for $2 \leq p<\infty$ for any sequence $\left(\nu_{n}\right)$ of complex numbers satisfying $\left|\nu_{n}\right| \leq 1$. Because the operators $D_{n}$ and $\sigma_{n}$ are selfadjoint, so is the operator $T_{\nu}$. Since $T_{\nu}$ is a bounded operators from $L^{p}$ to $L^{p}$ when $2 \leq p<\infty$, by duality, $T_{\nu}$ is a bounded operator from $L^{p}$ to $L^{p}$ when $1<p \leq 2$. 
Thus $T_{\nu}$ is bounded for all $1<p<\infty$. Further, the constant (although possibly not the best constant) can be taken independent of the sequence $\left(\nu_{n}\right)$. More precisely, for $1<p \leq 2$ we have (with $\frac{1}{p}+\frac{1}{q}=1$ )

$$
\begin{aligned}
\left\|T_{\nu} f\right\|_{p} & =\sup _{\|g\|_{q}=1} \int\left\{\sum_{k=1}^{\infty}\left(\sigma_{n_{k}} f(x)-D_{n_{k}} f(x)\right) \nu(k)\right\} g(x) d x \\
& =\sup _{\|g\|_{q}=1}\left\{\sum_{k=1}^{\infty} \int \sigma_{n_{k}} f(x) g(x) d x-\int D_{n_{k}} f(x) g(x) d x\right\} \nu(k) \\
& =\sup _{\|g\|_{q}=1}\left\{\sum_{k=1}^{\infty} \int f(x) \sigma_{n_{k}} g(x) d x-\int f(x) D_{n_{k}} g(x) d x\right\} \nu(k) \\
& =\sup _{\|g\|_{q}=1} \int f(x)\left\{\sum_{k=1}^{\infty}\left(\sigma_{n_{k}} g(x)-D_{n_{k}} g(x)\right) \nu(k)\right\} d x \\
& =\sup _{\|g\|_{q}=1} \int f(x) T_{\nu} g(x) d x \\
& \leq \sup _{\|g\|_{q}=1}\|f\|_{p}\left\|T_{\nu} g\right\|_{q} \\
& \leq c \sup _{\|g\|_{q}=1}\|f\|_{p}\|g\|_{q}=c\|f\| .
\end{aligned}
$$

Completion of the proof of Theorem 1.5 We already know the short variation operator is a bounded operator for all $p, 1<p<\infty$, because of Zygmund [10. Hence we need to show that $V_{\varrho}\left(\sigma_{2^{n}} f\right)$ is a bounded operator on $L^{p}$ for $1<p<\infty$. We have already shown that $\left(\sigma_{2^{n}}\right) \sim_{p}\left(D_{2^{n}}\right)$. From [2] we know $V_{\varrho}\left(D_{2^{n}} f\right)$ is a bounded operator on all $L^{p}, 1<p<\infty$. Thus, by Lemma 2.7 we know $V_{\varrho}\left(\sigma_{2^{n}} f\right)$ is bounded on all $L^{p}, 1<p<\infty$. We could also use the fact that $\left(\sigma_{2^{n}}\right) \sim_{p}\left(E_{n}\right)$, and apply Lepingle's result for martingales [5].

Remark 5.2. Before we go to Poisson kernel, we end this section by stating a general theorem whose proof is essentially given by arguments of the previous sections.

Theorem 5.3. Let $\left(T_{n}\right)$ be a family of integral operators on $L^{2}(\mathbb{T})$, with kernel $\left(W_{n}(x)\right)$ satisfying the following properties:

(1) There exists a constant $c>0$ such that the Fourier transform $\widehat{W}_{n}(j)$ satisfies

$$
\begin{array}{rlrl}
\left|1-\widehat{W}_{n}(j)\right| & \leq c \cdot|j / n|^{\sigma}, & & |j| \leq n, \\
\left|\widehat{W}_{n}(j)\right| \leq c \cdot|n / j|^{\sigma}, & & |j|>n,
\end{array}
$$

for some $\sigma>0$.

(2) There exists a constant $c>0$ such that the kernel $W_{n}(x)$ satisfies

$$
\left|W_{n}(x)\right| \leq \frac{c}{n x^{2}}
$$


(3) There exists a constant $c>0$ such that the difference of the kernel $W_{n}(x)-$ $W_{n}(y)$ satisfies

$$
\left|W_{n}(x)-W_{n}(y)\right| \leq \begin{cases}c n^{2}|x-y|, & \max \{n|x-y|, \min \{n|x|, n|y|\}\}<1 \\ c\left(\frac{|x-y|}{x^{2}}+\frac{|x-y|}{y^{2}}\right), & \min \{n|x|, n|y|\} \geq 1\end{cases}
$$

(4) The corresponding $G$-operator

$$
G_{T}(f)=\left(\sum_{n=1}^{\infty} n\left|T_{n} f(x)-T_{n-1} f(x)\right|^{2}\right)^{\frac{1}{2}}
$$

is bounded in $L^{p}, 2 \leq p<\infty$.

Then for $\varrho>2$ and $2 \leq p<\infty$

$$
\left\|V_{\varrho}(T f)\right\|_{p} \leq c(p, \varrho)\|f\|_{p} .
$$

If in additional, $\left(T_{n}\right)$ is self-adjoint, and (4) holds for $1<p<\infty$, then we also have

$$
\left\|V_{\varrho}(T f)\right\|_{p} \leq c(p, \varrho)\|f\|_{p}
$$

when $1<p \leq 2$ and $\varrho>2$.

Proof. Condition (11) and Lemma 2.8 show that $\left(T_{2^{n}}\right) \sim_{2}\left(D_{2^{n}}\right)$. By Remark 2.9 $\left(D_{2^{n}}\right) \sim_{2}\left(E_{n}\right)$. Thus, $\left(T_{2^{n}}\right) \sim_{2}\left(E_{n}\right)$. We know that $V_{\varrho}\left(E_{n}\right)$ is bounded in $L^{2}$ by Lepingle's Theorem for martingales [5]. Thus by Lemma 2.7] $V_{\varrho}\left(T_{2^{n}}\right)$ is bounded in $L^{2}$. Conditions (2) and (3), Lemma 4.5, and Theorem 4.1 shows that $V_{\varrho}\left(T_{2^{n}}\right)$ is bounded from $L^{\infty}$ to BMO. Thus, by interpolation, $V_{\varrho}\left(T_{2^{n}}\right)$ is bounded in $L^{p}$, $2 \leq p<\infty$. Condition (4) and the fact that $V_{\varrho}^{S}(T) \leq c G_{T}$ as shown virtually in Section 3.2 implies that $V_{\varrho}^{S}(T)$ is bounded in $L^{p}, 2 \leq p<\infty$. Thus, $V_{\varrho}(T)$ is bounded in $L^{p}, 2 \leq p<\infty$. In addition, the linear operators between $\left(T_{2^{n}}\right)$ and $\left(E_{n}\right)$ defined similarly to those in Section 4 are also bounded in $L^{2}$ and from $L^{\infty}$ to $\mathrm{BMO}$, and hence in $L^{p}, 2 \leq p<\infty$. Namely, $\left(T_{2^{n}}\right) \sim_{p}\left(E_{n}\right), 2 \leq p<\infty$. Thus, we can handle the case $1<p \leq 2$ by the same arguments given in the beginning of this section by using duality arguments. First, by duality, $\left(T_{2^{n}}\right) \sim_{p}\left(E_{n}\right), 1<p \leq 2$. So by Lemma 2.7 and Lepingle's result which says $V_{\varrho}\left(E_{n}\right)$ is bounded in $L^{p}, 1<p<\infty$, we have $V_{\varrho}\left(T_{2^{n}}\right)$ is bounded in $L^{p}, 1<p \leq 2$. Next, condition (4) and the fact that $V_{\varrho}^{S}(T) \leq c G_{T}$ show that $V_{\varrho}^{S}(T)$ is bounded in $L^{p}, 2 \leq p<\infty$. Therefore, $V_{\varrho}(T)$ is bounded in $L^{p}, 1<p \leq 2$.

Note if only conditions (1)-(3) hold, then we have that the "long variation" $V_{\varrho}\left(T_{2^{n}} f\right)$ is bounded in $L^{p}, 2 \leq p<\infty$, and bounded in $L^{p}, 1<p<\infty$, if $T_{n}$ is also self-adjoint.

\section{The PoIsson Kernel}

The results in this section parallel those in the earlier sections for the Fejér kernel. We will verify that conditions (11), (2), (3) of Theorem 5.3 hold for $\left(P_{r_{n}}\right)$ where $r_{n}=1-\frac{1}{n}$. Thus, the corresponding "long variation" of Poisson operator $V_{\varrho}\left(P_{r_{2} n} f\right)$ is bounded in $L^{p}, 1<p<\infty$. We will see that the short variation can be handled by an operator studied by Zygmund and is bounded in $L^{p}, 1<p<\infty$. Thus, by the triangle inequality, $V_{\varrho}\left(P_{r} f\right)$ is bounded in $L^{p}, 1<p<\infty$. 
Theorem 6.1. If $r_{n}=1-\frac{1}{n}$, then $V_{\varrho}\left(P_{r_{2} n} f\right)$ is a bounded operator on $L^{p}, 1<$ $p<\infty$.

Proof. It is enough to verify that the family of operators $\left(T_{n}\right)=\left(P_{1-\frac{1}{n}}\right)$ satisfies conditions (11), (21), (31) of Theorem 5.3.

First note the kernel of $T_{n}$ is

$$
\begin{aligned}
W_{n}(x)=P_{1-\frac{1}{n}}(x) & =1+2 \sum_{j=1}^{\infty}\left(1-\frac{1}{n}\right)^{j} \cos j x \\
& =\frac{1-\left(1-\frac{1}{n}\right)^{2}}{1-2\left(1-\frac{1}{n}\right) \cos x+\left(1-\frac{1}{n}\right)^{2}} \\
& =\frac{\frac{2}{n}-\frac{1}{n^{2}}}{4\left(1-\frac{1}{n}\right) \sin ^{2}\left(\frac{x}{2}\right)+\frac{1}{n^{2}}}
\end{aligned}
$$

and

$$
\widehat{W}_{n}(j)=\left(1-\frac{1}{n}\right)^{|j|}
$$

Clearly,

$$
W_{n}(x) \leq \frac{c}{n x^{2}} .
$$

Thus condition (2) holds. Next, since $1-x \leq e^{-x}$ when $x \geq 0$, for $n<|j|$ we have $\left(1-\frac{1}{n}\right)^{|j|} \leq e^{-|j| / n} \leq \frac{n}{|j|}$. Note also by calculus,

$$
1-(1-x)^{r} \leq r x
$$

when $0 \leq x \leq 1$ and $r \geq 1$. The above inequality also holds when $r=0$. Consequently, for $|j| \leq n$, we have

$$
\left|1-\left(1-\frac{1}{n}\right)^{|j|}\right| \leq|j| / n
$$

Hence condition (1) holds. To verify condition (3), note

$$
\begin{aligned}
W_{n}(x)-W_{n}(y) & =\frac{4\left(1-\frac{1}{n}\right)\left(\frac{2}{n}-\frac{1}{n^{2}}\right)\left(\sin ^{2}\left(\frac{x}{2}\right)-\sin ^{2}\left(\frac{y}{2}\right)\right)}{\left(4\left(1-\frac{1}{n}\right) \sin ^{2}\left(\frac{x}{2}\right)+\frac{1}{n^{2}}\right)\left(4\left(1-\frac{1}{n}\right) \sin ^{2}\left(\frac{y}{2}\right)+\frac{1}{n^{2}}\right)} \\
& =-\frac{4\left(1-\frac{1}{n}\right)\left(\frac{2}{n}-\frac{1}{n^{2}}\right) \sin \left(\frac{x+y}{2}\right) \sin \left(\frac{x-y}{2}\right)}{\left(4\left(1-\frac{1}{n}\right) \sin ^{2}\left(\frac{x}{2}\right)+\frac{1}{n^{2}}\right)\left(4\left(1-\frac{1}{n}\right) \sin ^{2}\left(\frac{y}{2}\right)+\frac{1}{n^{2}}\right)} .
\end{aligned}
$$

Thus, when $\max \{n|x-y|, \min \{n|x|, n|y|\}\}<1$, we have $n|x|<2$ and $n|y|<2$. So

$$
\left|W_{n}(x)-W_{n}(y)\right| \leq c n^{4} \frac{1}{n}|x+y||x-y| \leq c n^{2}|x-y| .
$$

If $\min \{n|x|, n|y|\} \geq 1$, then $\frac{1}{n} \leq|x|, \frac{1}{n} \leq|y|$. So

$$
\left|W_{n}(x)-W_{n}(y)\right| \leq \frac{c(|x|+|y|)^{2}|x-y|}{x^{2} y^{2}} \leq c\left(\frac{1}{x^{2}}+\frac{1}{y^{2}}\right)|x-y| .
$$

This verifies (3). 
We now need to consider the short variation. Let $I_{k}=\left[1-1 / 2^{k}, 1-1 / 2^{k+1}\right)$, and define the short variation operator by

$$
V_{\varrho}^{S}\left(P_{r} f\right)(x)=\sup _{\left(s_{n}\right) \nearrow^{-}} \sum_{k=1}^{\infty}\left(\sum_{n:\left(s_{n}, s_{n+1}\right) \subset I_{k}}\left|P_{s_{n}} f(x)-P_{s_{n+1}} f(x)\right|^{2}\right)^{\frac{1}{2}} .
$$

Let

$$
V_{\varrho, k}^{S}\left(P_{r} f\right)(x)=\sup _{\left(s_{n}\right) \nearrow 1^{-}}\left(\sum_{n:\left(s_{n}, s_{n+1}\right) \subset I_{k}}\left|P_{s_{n}} f(x)-P_{s_{n+1}} f(x)\right|^{2}\right)^{\frac{1}{2}} .
$$

We have

$$
\left(V_{\varrho}^{S}\left(P_{r} f\right)(x)\right)^{2} \leq \sum_{k}\left(V_{\varrho, k}^{S}\left(P_{r} f\right)(x)\right)^{2} .
$$

We will first get an estimate of $V_{\varrho, k}^{S}\left(P_{r} f\right)(x)$. Fix $x$ and a sequence $\left(s_{n}\right) \nearrow 1^{-}$. Let $A_{k}=\left\{n:\left(s_{n}, s_{n+1}\right) \subset I_{k}\right\}$.

We know that

$$
\left|P_{s_{n}} f(x)-P_{s_{n+1}} f(x)\right|=\left|\int_{s_{n}}^{s_{n+1}} \frac{\partial}{\partial r} P_{r} f(x) d r\right| \leq \int_{s_{n}}^{s_{n+1}}\left|\frac{\partial}{\partial r} P_{r} f(x)\right| d r .
$$

Hence we have

$$
\begin{aligned}
V_{\varrho, k}^{S}\left(P_{r} f\right)(x) & \leq\left(\sum_{n \in A_{k}}\left[\int_{s_{n}}^{s_{n+1}}\left|\frac{\partial}{\partial r} P_{r} f(x)\right| d r\right]^{2}\right)^{\frac{1}{2}} \\
& =\left(\sum_{n \in A_{k}}\left[\int_{s_{n}}^{s_{n+1}}\left|\frac{\partial}{\partial r} P_{r} f(x)\right| \chi_{\left[s_{n}, s_{n+1}\right]}(r) d r\right]^{2}\right)^{\frac{1}{2}} \\
& =\left(\sum_{n \in A_{k}}\left[\int_{I_{k}}\left|\frac{\partial}{\partial r} P_{r} f(x)\right| \chi_{\left[s_{n}, s_{n+1}\right]}(r) d r\right]^{2}\right)^{\frac{1}{2}} \\
& \leq\left(\int_{I_{k}}\left|\frac{\partial}{\partial r} P_{r} f(x)\right|^{2} \sum_{n \in A_{k}}\left(s_{n+1}-s_{n}\right)\right)^{\frac{1}{2}} d r \\
& \leq\left(\left|I_{k}\right|\right)^{\frac{1}{2}}\left(\int_{I_{k}}\left|\frac{\partial}{\partial r} P_{r} f(x)\right|^{2} d r\right)^{\frac{1}{2}} \\
& =\left(\int_{I_{k}}\left|I_{k}\right|\left|\frac{\partial}{\partial r} P_{r} f(x)\right|^{2} d r\right)^{\frac{1}{2}} \\
& \leq 2\left(\int_{I_{k}}(1-r)\left|\frac{\partial}{\partial r} P_{r} f(x)\right|^{2} d r\right)^{\frac{1}{2}}
\end{aligned}
$$

Consequently the square of the short variation is dominated by a constant multiple of

$$
\sum_{k} \int_{I_{k}}(1-r)\left|\frac{\partial}{\partial r} \operatorname{Pf}(r, x)\right|^{2} d r \leq \int_{0}^{1}(1-r)\left|\frac{\partial}{\partial r} \operatorname{Pf}(r, x)\right|^{2} d r=G f(x)^{2} .
$$

Since $G f(x)$ is a bounded operator on $L^{p}, 2 \leq p<\infty$, so is the short variation operator.

With the $G$-operator in condition (4) of Theorem 5.3 replaced by $G f(x)$ above and an application of Theorem 5.3 we get the following. 
Theorem 6.2. The variation operator

$$
V_{\varrho}(P f)(x)=\left(\sup _{\left(s_{n}\right) \nearrow^{-}} \sum_{n}\left|P_{s_{n}} f(x)-P_{s_{n+1}} f(x)\right|^{\varrho}\right)^{\frac{1}{\varrho}}
$$

is a bounded operator on $L^{p}$ for $1<p<\infty$.

\section{JUMP INEQUALITIES}

We can now give the proofs of Theorems 1.6 and 1.8. We only give the details for the proof of Theorem 1.6] since the details are similar in both cases.

Proof of Theorems [1.6] and [1.8. Let $B_{k} f(x)=\sigma_{2^{n}} f(x)$ if $2^{n} \leq k<2^{n+1}, k=$ $1,2, \ldots$ Then we have

$$
\Lambda\left(\left(\sigma_{n}\right), f, \lambda\right)(x) \leq \Lambda\left(\left(\sigma_{n}-B_{n}\right), f, \frac{\lambda}{2}\right)(x)+\Lambda\left(\left(B_{n}\right), f, \frac{\lambda}{2}\right)(x) .
$$

For the first term on the right-hand side we note that

$$
\frac{\lambda}{2} \sqrt{\Lambda\left(\left(\sigma_{n}-B_{n}\right), f, \frac{\lambda}{2}\right)(x)} \leq V_{2}\left(\left(\sigma_{n}-B_{n}\right) f\right)(x)
$$

and this is just the short variation operator which we have already shown to be bounded on all $L^{p}, 1<p<\infty$. The second term on the right-hand side is the same as $\Lambda\left(\left(\sigma_{2^{n}}\right), f, \frac{\lambda}{2}\right)(x)$. We have established that $\left(\sigma_{2^{n}}\right) \sim_{p}\left(D_{2^{n}}\right)$ and in [2] we saw that $\left\|\sqrt{\Lambda\left(\left(D_{2^{n}}\right), f, \lambda\right)}\right\|_{p} \leq \frac{c}{\lambda}\|f\|_{p}$. (We could also note that $\left(\sigma_{2^{n}}\right) \sim_{p}\left(E_{n}\right)$ and apply the well-known result for martingale jumps.) In either case the result now follows by applying Lemma 2.7. Theorem 1.8 follows in the same way.

\section{Negative Results}

In the above results regarding variation operators we always assumed that $\varrho>2$. It is natural to ask if this is necessary or just an artifact of the proof. In this section we prove Theorems 1.12 and 1.13 stated in Section 1 showing that in fact $\varrho>2$ is necessary.

Both of these results follow from the analogous theorem for dyadic martingales. To see that this is the case, assume for now that we have the following proposition.

Proposition 8.1. There is a function $f \in L^{\infty}(\mathbb{T})$ such that $V_{2}\left(E_{n} f\right)(x)=\infty$ a.e.

Proof of Theorems 1.12 and 1.13. Assuming Proposition 8.1. we immediately obtain Theorem 1.12 by using Lemma 2.7, Remark 2.9 and Theorem 3.1 .

The same argument works for the Poisson kernel and proves Theorem 1.13

Before we can make the construction required to prove Proposition 8.1 we need some preliminary results that were obtained by Qian [6].

For a sequence of random variables, $Y_{i}$, let $S_{k}=\sum_{i=1}^{k} Y_{i}$ and let

$$
V_{\varrho}^{n}\left(S_{k}\right)\left(=V_{\varrho}^{n}\left(\left(S_{k}\right)\right)\right)=\sup _{\left(n_{i}\right) \nearrow,\left(n_{i}\right) \leq n}\left(\sum_{i=1}^{\infty}\left|S_{n_{i}}-S_{n_{i+1}}\right|^{\varrho}\right)^{\frac{1}{\varrho}} .
$$

We will abbreviate $\log \log n$ by $L L n$.

Theorem 8.2 (Qian). Let $\left(Y_{i}\right)$ be i.i.d. with $E Y_{1}=0, E\left(Y_{1}^{2}\right)=1$ and $E\left(\left|Y_{1}\right|^{1+\delta}\right)<$ $\infty$ for some $\delta>0$. Then for any $c \in\left(0, \frac{1}{12}\right)$ we have $P\left(V_{2}^{n}\left(S_{k}\right)>c \sqrt{n L L n}\right) \rightarrow 1$ as $n \rightarrow \infty$.

Proof of Proposition 8.1. The proof of this will depend on the following lemma. 
Lemma 8.3. Let $N_{0}, L$, and $\epsilon>0$ be given. Then there is a function $f \in L^{\infty}(\mathbb{T})$ and an integer $N$ such that

(1) $\|f\|_{\infty} \leq 1$,

(2) $E_{k}(f)=0$ for all $k \leq N_{0}$,

(3) $f$ is measurable with respect to the $\sigma$-field $\mathcal{F}_{N_{0}+N}$,

(4) $V_{2}\left(E_{n} f\right)(x) \geq L$ except possibly on a set of measure less than $\epsilon$.

Proof. Let $\left(r_{k}\right)$ denote the sequence of Rademacher functions. Let $f_{n}=\sum_{k=N_{0}}^{N_{0}+n} r_{k}$. Note that $E_{n_{i}} f_{n}=f_{n_{i}}$ if $N_{0} \leq n_{i} \leq N_{0}+n$. From Qian's result, taking $c=1 / 20$, we know that given $\epsilon>0$, if $N$ is large enough, then we have

$$
P\left(\sup _{\left(n_{i}\right) \nearrow,\left(n_{i}\right) \leq N}\left(\sum_{i=1}^{\infty}\left|E_{n_{i}} f_{N}(x)-E_{n_{i+1}} f_{N}(x)\right|^{2}\right)^{\frac{1}{2}}>\frac{1}{20} \sqrt{N L L N}\right)>1-\frac{\epsilon}{2} .
$$

Increase $N$, if necessary, so that

$$
\frac{\log \log \log N}{\log \log N}<\epsilon \text { and } \sqrt{\log \log \log N}>100 L
$$

Take

$$
M=2[\sqrt{N / \epsilon}]-1 .
$$

Define the stopping time $\tau$ by $\tau=\inf \left\{n:\left|f_{n}\right| \geq M\right\}$. Let $f_{n}^{\tau}$ denote the martingale stopped at time $\tau$. Then

$$
\begin{aligned}
P(\tau<N) & <\int_{\{\tau<N\}}\left(\frac{f_{N}^{\tau}}{M+1}\right)^{2} \\
& \leq \frac{1}{(M+1)^{2}} E\left(\left|f_{N}^{\tau}\right|^{2}\right) \\
& \leq \frac{1}{(M+1)^{2}} E\left(\left|f_{N}\right|^{2}\right) \\
& \leq \frac{N}{(M+1)^{2}} .
\end{aligned}
$$

Using our choice of $N$ and $M$, we see that $P(\tau<N)<\frac{\epsilon}{2}$.

We now have

$$
\begin{aligned}
& P\left(\sup _{\left(n_{i}\right) \nearrow,\left(n_{i}\right)<N}\left(\sum_{i=1}^{\infty}\left|f_{n_{i}}^{\tau}-f_{n_{i+1}}^{\tau}\right|^{2}\right)^{\frac{1}{2}}>\frac{1}{20} \sqrt{N L L N}\right) \\
& \quad \geq P\left(\sup _{\left(n_{i}\right) \nearrow,\left(n_{i}\right)<N}\left(\sum_{i=1}^{\infty}\left|f_{n_{i}}-f_{n_{i+1}}\right|^{2}\right)^{\frac{1}{2}}>\frac{1}{20} \sqrt{N L L N}, \tau \geq N\right) \\
& \quad \geq P\left(\sup _{\left(n_{i}\right) \nearrow,\left(n_{i}\right)<N}\left(\sum_{i=1}^{\infty}\left|f_{n_{i}}-f_{n_{i+1}}\right|^{2}\right)^{\frac{1}{2}}>\frac{1}{20} \sqrt{N L L N}\right)-P(\tau<N) \\
& \quad \geq 1-\frac{\epsilon}{2}-\frac{\epsilon}{2}=1-\epsilon .
\end{aligned}
$$

Now let $g_{n}=\frac{1}{M+1} f_{n}^{\tau}$. Then $\left\|g_{n}\right\|_{\infty} \leq 1$ and

$$
P\left(\sup _{\left(n_{i}\right) \nearrow,\left(n_{i}\right)<N}\left(\sum_{i=1}^{\infty}\left|g_{n_{i}}-g_{n_{i+1}}\right|^{2}\right)^{\frac{1}{2}}>\frac{1}{M+1} \frac{1}{20} \sqrt{N L L N}\right) \geq 1-\epsilon .
$$


Consequently we have

$$
P\left(\sup _{\left(n_{i}\right) \nearrow,\left(n_{i}\right)<N}\left(\sum_{i=1}^{\infty}\left|g_{n_{i}}-g_{n_{i+1}}\right|^{2}\right)^{\frac{1}{2}}>\frac{1}{100} \sqrt{\log \log \log N}\right)>1-\epsilon .
$$

Since $N$ satisfies $\sqrt{\log \log \log N}>100 L$ the proof is complete.

We now need to use the lemma to put together pieces as done in 11 to finish the proof of Proposition 8.1 First assume that $N_{0}=0$. Let $L=100 \times 2^{1}$ and $\epsilon=\frac{1}{2^{1}}$. Let $f$ be the function obtained by Lemma 8.3 , and define $g_{1}=\frac{1}{2} f$. Let $b_{1}=g_{1}$. Denote by $N_{1}$ the integer $N$ obtained by Lemma 8.3. We then have $V_{2}\left(b_{1}\right) \geq 100$ for $x$ in a set $X_{1} \subset X$ of measure greater than $1-\frac{1}{2}$. We now take $N_{0}$ in Lemma 8.3 to be $N_{1}$. We take $L=100^{2} \times 2^{2}$ and $\epsilon=\frac{1}{2^{2}}$. Let $f$ be the function obtained by Lemma 8.3 and define $g_{2}=\frac{1}{2^{2}} f$. Since $E_{n} f=0$ for $0 \leq n \leq N_{0}$, and $E_{n} g_{1}=g_{1}$ for all $n \geq N_{0}$, if we define $b_{2}=g_{1}+g_{2}$, we see that $V_{2}\left(b_{2}\right) \geq 100^{2}$ for $x \in X_{2} \subset X$ with measure at least $1-\frac{1}{2^{2}}$. We repeat the construction, so that at the $k$ th stage we have $N_{0}=N_{1}+N_{2}+\cdots+N_{k-1}$. We have $b_{k-1}$ measurable with respect to $\mathcal{F}_{N_{0}}$, and $V_{2} b_{k-1}>100^{k-1}$ on $X_{k-1} \subset X$ with $\mu\left(X_{k-1}\right)>1-\frac{1}{2^{k-1}}$. We take $L=100^{k} 2^{k}$ and $\epsilon=\frac{1}{2^{k}}$. We construct the function $f$ using Lemma 8.3 and define $g_{k}$ to be $\frac{1}{2^{k}} f$. We let $b_{k}=b_{k-1}+g_{k}$. Hence $V_{2} b_{k}>100^{k}$ on a set $X_{k} \subset X$ of measure greater than $1-\frac{1}{2^{k}}$. At each stage $\left\|g_{k}\right\|_{\infty} \leq \frac{1}{2^{k}}$ so $\sum_{k} g_{k}$ converges. Let $b=\sum_{k} g_{k}$. Then $\|b\|_{\infty} \leq 1$ and for all $k V_{2} b(x) \geq V_{2}\left(b_{k}\right) \geq 100^{k}$ on a set of measure at least $1-\frac{1}{2^{k}}$. Since $k$ was arbitrary, we are done.

\section{Differentiation}

Recall that $D_{2^{n}} f(x)=\frac{2^{n}}{2 \pi} \int_{\frac{-\pi}{2^{n}}}^{\frac{\pi}{2^{n}}} f(x+t) d t$. We conclude this section with a negative result about the variation for the Lebesgue differentiation operator. The positive result obtained in [2] was

Theorem 9.1. For $\varrho>2$ the operator $V_{\varrho}(D f)$ is bounded on all $L^{p}, 1<p<\infty$, and is weak type $(1,1)$.

Using the above techniques, we now have the following theorem.

Theorem 9.2. There is an $f \in L^{\infty}$ such that $V_{2}(D f)(x)=\infty$ a.e.

Proof. Apply Proposition 8.1, Remark 2.9, and Lemma 2.7.

\section{ACKNOWLEDGMENT}

We would like to thank Mustafa Akcoglu, Joseph Rosenblatt and Mate Wierdl for several interesting conversations related to the contents of this paper. We would also like to thank the referee for several comments that greatly improved the exposition of the paper.

\section{REFERENCES}

[1] Akcoglu, M., Jones, R. and Schwartz, P., Variation in probability, ergodic thoery and analysis, Illinois J. of Math., 42 (1998) 154-177. MR 99a:60048

[2] Jones, R., Kaufman, R., Rosenblatt, J., and Wierdl, M., Oscillation in ergodic theory, Ergodic Theory and Dynam. Sys. 18 (1998) 889-935. MR 2000b:28019

[3] Jones, R.L. and Rosenblatt, J., Differential and ergodic transforms, Mathematische Annalen, 323 (2002) 525-546. MR 2003g:37003 
[4] Kaczmarz, S., Über die Konvergenz der Reihen von Orthogonal-funktionen, Math. Z., 23 (1925) 263-270.

[5] D. Lepingle, La variation d'order $p$ des semi-martingales, Z. Wahrscheinlichkeitstheorie verw. Gebiete 36 (1976) 295-316. MR 54:8849

[6] J. Qian, The p variation of partial sum processes and the emperical process, Ann. of Prob., 26 (1998) 1370-1383. MR 99i:60052

[7] E. M. Stein, The development of square funtions in the work of A. Zygmund Bull. Amer. Math. Soc. (N.S.) 7 (1982), no. 2, 359-376. MR 83i:42001

[8] E. M. Stein, Singular Integrals and Differentiablity Properties of Functions Princeton University Press, Princeton, N.J., 1970. MR 44:7280

[9] Zygmund, A., Une remarque sur un théorème de M. Kaczmarz Math. Z., 25 (1926) 297-298.

[10] A. Zygmund, Trigonometric series, Vol. 2, second edition, Cambridge University Press, New York, 1977. MR 58:29731

Department of Mathematics, DePaul University, 2320 N. Kenmore, Chicago, Illinois 60614

E-mail address: rjones@condor.depaul.edu

Department of Mathematics, DePaul University, 2320 N. Kenmore, Chicago, Illinois 60614

E-mail address: gwang@condor.depaul.edu 